\title{
IMMUNOTHERAPY
}

\section{New or second CARs for ALL}

Anti-CD19 chimeric antigen receptor (CAR) T cells have remarkable clinical activity against B-cell acute lymphoblastic leukaemia (B-ALL); indeed, the first approval of such agents was for this disease. Nevertheless, relapses can occur, often owing to emergence of CD19- leukaemic blasts. Novel CAR T cells targeting CD22, expression of which is usually retained after anti-CD19 immunotherapy, have now been evaluated in a phase I trial.

Terry Fry et al. treated 21 children or adults with relapsed and/or refractory B-ALL with autologous anti-CD22 CAR T cells. Notably, 17 patients had received prior anti-CD19 therapy (15 with CAR T cells), and 10 had $\mathrm{CD}^{-} 9^{-}$or CD19 ${ }^{\mathrm{dim}}$ blasts. The complete remission (CR) rate among 15 patients who received $\geq 1 \times 10^{6}$ anti-CD 22 CAR T cells per $\mathrm{kg}$ was $73 \%$ - comparable to that reported with anti-CD19 CAR T cells. All five patients with $\mathrm{CD} 19^{-}$or $\mathrm{CD} 19^{\text {dim }}$ disease treated at this dose achieved a CR. The median duration of remission was 6 months; however, one CR was ongoing at 21 months. As with other CAR-T-cell therapies, cytokine-release syndrome was common, but manageable.
"Our findings demonstrate, for the first time, that targeting a second antigen can overcome immunotherapeutic resistance," Fry explains. "We also demonstrated a novel form of immune escape caused by a decrease in target antigen density [CD22] on cancer cells, rather than complete loss of that antigen as has been seen with CD19," he adds.

"With a novel second target for effective CAR-T-cell therapy, but similar issues with antigen-negative escape, the goal is to develop multiantigen-targeting strategies in order to reduce the likelihood of relapse - similar to the paradigm of multiagent chemotherapy," states co-author Nirali Shah. Indeed, the investigators showed that bispecific anti-CD19/ CD22 CAR T cells have promising activity against B-ALL cell lines with mixed CD19/ CD22 expression and patient-derived xenografts. "We are currently conducting clinical trials testing this approach," Fry concludes.

David Killock

ORIGINAL ARTICLE Fry, T. J. et al. CD22-targeted CAR T cells induce remission in B-ALL that is naive or resistant to CD19targeted CAR immunotherapy. Nat. Med. http://dx.doi.org/ $10.1038 / \mathrm{nm} .4441$ (2017) 